Thorax, 1977, 32, 53-59

\title{
Further observations on luminal deformity and stenosis of nonrespiratory bronchioles in pulmonary emphysema
}

\author{
ALENA LINHARTOVÁ, A. E. ANDERSON, JR., AND ALVAN G. FORAKER \\ From Sikl's Department of Pathology, Charles University, Plzeñ, Czechoslovakia and the Research \\ Department, Baptist Memorial Hospital, 800 Prudential Drive, Jacksonville, Florida, USA
}

Linhartová, Alena, Anderson, A. E., and Foraker, A. G. (1977). Thorax, 32, 53-59. Further observations on luminal deformity and stenosis of nonrespiratory bronchioles in pulmonary emphysema. In an endeavour to elucidate the anatomical basis for the increased resistance to airflow which characterises the most peripheral conducting air passages in pulmonary emphysema, lumina of nonrespiratory bronchioles of lungs with minly centrilobular disease were assessed for two- and three-dimensional features by: (1) determination of percentage conformity of the lumina of individual bronchioles in histological sections to hypothetical planes through cylinders (ie, ellipses with the same areas and circumferences), and (2) comparison of luminal areas at regular intervals along bronchiolar longitudinal axes.

The lumina of most nonrespiratory bronchioles from normal lungs conformed closely to their respective ellipses, thus corroborating previous observations that they are normally cylindroid. In contrast, there was a substantial excess of plane section deformities in the lumina of nonrespiratory bronchioles from the emphysematous specimens. The incidence of stenotic bronchioles (by both diameter and area determinations) was also greatly increased in emphysema. Since there was a strong positive association between such stenotic lesions and bronchiolar deformity, the latter was concluded to be a major factor in bronchiolar restriction. Furthermore, these characteristics seemed to have three-dimensional expression, for the lumina of stenotic bronchioles in emphysema were irregular in a longitudinal fashion.

A number of investigations have demonstrated widespread stenosis of the nonrespiratory bronchioles in pulmonary emphysema (Spain and Kaufman, 1953; Anderson and Foraker, 1962; Linhartová et al., 1971; Depierre et al., 1972; Matsuba and Thurlbeck, 1972). This provides one anatomical explanation for the increased flow resistance shown to be characteristic of the disease at this level of the conduction system (Campbell et al., 1957; Hogg et al., 1968; Silvers et al., 1972). In an earlier effort to elucidate the nature of this stenosis in our laboratories, histological sections of nonrespiratory bronchioles were graphically assessed for luminal deformities according to their location by circumference and area parameters in a mathematical system of parabolas (Linhartová et al., 1973). This approach was based on the principle that a circle constitutes the most compact shape for a given circumference, and variation from this form in cross-sectional contours would be a potential basis for flow restriction. The lumina of the bronchioles of emphysematous lungs did, indeed, deviate excessively from the parabola for an ideal circular configuration in that analysis. On the other hand. sections of bronchioles from normal lungs demonstrated similar changes of lesser degree. This was thought to be at least partially an effect of inadvertent oblique histological sectioning, and a comparable artifact was evidently present in the emphysematous samples. The relative significance of oblique sectioning, however, was largely conjectural. Later, a series of models of bronchioles which were constructed from magnified serial histological sections seemed to confirm the presence of deformed shapes in emphysema (Lin- 
hartová et al., 1974). However, the number of airways which could be studied with this approach was of necessity small, thus introducing a potentially significant sampling problem. Type of disease was not considered in any of the previous studies. In an endeavour to compensate for these presumed deficiencies and evaluate bronchiolar stenosis in the present investigation, nonrespiratory bronchioles from subjects with normal lungs and known variety of emphysema were analysed individually in histological sections with a series of ellipses. In selected instances, nonrespiratory bronchioles with restrictions were also evaluated for longitudinal irregularities.

\section{Material and methods}

A fundamental concept of this investigation is that the lumina of the conducting air passages of lungs are normally cylindroid and straight between branchings. This may be affirmed for all but the very last generations of branchings by simple inspection of bronchograms in various projections. Moreover, observations on serial section reconstructions give no indication of any variation in the most peripheral ramifications of the conducting system from this arrangement, which permits maximum conductance (Linhartová et al., 1974). It follows that the lumina of most nonrespiratory bronchioles of inflated, excised normal lungs should be closely circular in a plane perpendicular to their long axes and by ellipsoids of varying diameters in oblique projections. The following mathematical reasoning is based on these assumptions.

The relations between areas and circumferences of plane shapes, such as circles and ellipses which reflect different projections through a cylinder, produce values which are located along specific parabolas as size varies (Linhartová et al., 1973). Conversely, ellipses derived by correlation of circumference and area parameters of sections through lumina of given nonrespiratory bronchioles between branchings would be expected to coincide in reasonably close fashion with the actual lumina for the respective bronchioles if they were normal, ie, cylindroid. If they do not, deformities exist. The portion of a nonrespiratory bronchiole falling outside the limits of its ellipse reflects this deformity, and that portion of the nonrespiratory bronchiole within the confines of the ellipse gives an indication of conformity to the cylindroid shape. Conformity in such a system then may be expressed as a percentage in the following fashion:

$$
\text { Index of conformity }=\frac{\text { Identical area }}{\text { Bronchiolar area }} \times 100
$$

Histological sections of 374 nonrespiratorys bronchioles were studied. These were identified from randomly selected hematoxylin and eosirp stained parenchymal preparations of inflation-fixed lungs of normal and emphysematous subjects (in increments of 0 to 6). Bronchioles sectione longitudinally with divisions and incomplete walls were excluded. There was a total of 70 subject氮 (10 per increment category) and a mean of $5 \cdot 3 \mathrm{w}$ nonrespiratory bronchioles per case. Emphysema. severity and type were graded in paper whole lungi mounts (Gough and Wentworth, 1960), and the luminal circumferences and areas of bronchioles? were measured at levels of epithelial basement membranes from sketches of projected images (X100) as previously described (Linhartová et al.స్ 1971). From these circumference and aree measurements cardboard ellipses with correspond $\overrightarrow{0}$ ing parameters were constructed and cut out for the bronchioles. The ellipses were positioned over the respective sketches of the bronchioles in suclo a way that the area shared by both figures seemed maximal (Fig. 1). Areas were determined witlo planimetry, and degree of bronchiolar conformit昂 to the hypothetical sections through cylinders was

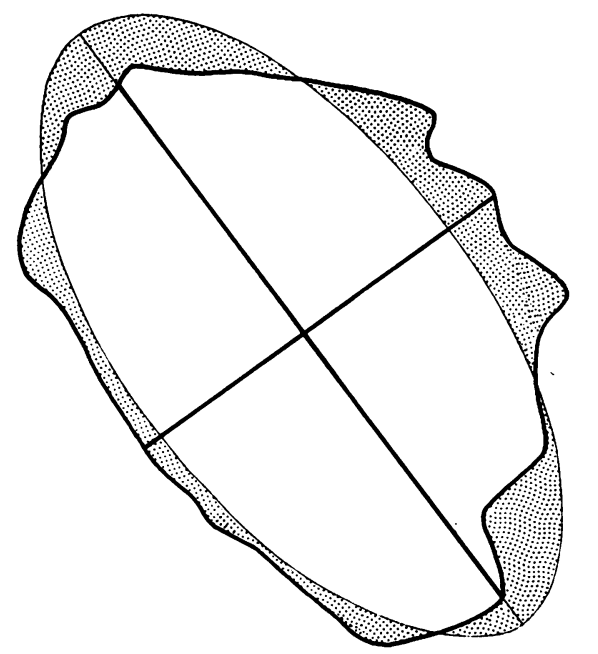

Fig. 1 Replica of a nonrespiratory bronchiolar lument (thick line) from histological section of normal lung and ellipse (thin line) with same area and circumference. Drawings are superimposed to share maximum area. Unstippled area here comprises $89 \%$ of the total area of the bronchiolar lumen and is a function of its conformity to the ellipsoidal shape (see text for details). 
expressed as a percentage in accordance with the aforementioned formula. Superimposition of ellipses on bronchiolar lumina also provided a standardised setting for measuring luminal diameters. These were taken as the bronchiolar diameters which coincided with the short axes of ellipses (Fig. 1). A preliminary tabulation seemed to indicate a natural break in the degree of conformity of bronchioles from the 30 lungs with no or minimal emphysema (grades $0-2$ with our grading system) and the 40 frankly emphysematous lungs (grades 3-6). These two arbitrary categories of specimens were therefore separated for statistical comparisons. For convenience, cases with no or minimal emphysema are listed simply as 'normal' under Results.

In an ancillary exercise, graphic representation of three-dimensional characteristics of bronchiolar restrictions in pulmonary emphysema was assessed from material used in a previous study of serial section reproductions (Linhartová et al., 1974). This was done by comparing luminal areas of 29 segments (15 from emphysematous lungs and 14 from normal lungs) of 21 nonrespiratory bronchioles in the smaller size categories at regular intervals, usually every 20th section (corresponding to increments of 120 microns). A single air passage was examined at every 30 th section (180 micron increments). Again, only segments of bronchioles uncomplicated by branchings were studied.

\section{Results}

It may be seen in Table 1 that this was essentially a study of changes occurring in the nonrespiratory bronchioles in centrilobular emphysema. Only two of the 40 lungs with unequivocal emphysema were judged to be exclusively of the panlobular type, and five contained approximately equal amounts of the two forms of disease. Most of the cases having panlobular emphysema were in lower severity categories.

Table 1 Classification of lungs with unequivocal emphysema by type and severity of disease ${ }^{t}$

\begin{tabular}{lrrrrrr}
\hline Type & \multicolumn{3}{l}{ Severity } & grade & & Total \\
\cline { 2 - 7 } & 3 & 4 & 5 & 6 & \\
\hline Centrilobular & 6 & 7 & 9 & 10 & 32 \\
Panlobular & 2 & - & - & - & $\frac{3}{2}$ \\
Equal & 2 & 3 & 1 & - & 6 \\
Total & 10 & 10 & 10 & 10 & 40 \\
\hline
\end{tabular}

${ }^{1}$ In Gough-Wentworth paper whole lung mounts.
The frequency distributions by percentage conformity of the lumina of nonrespiratory bronchioles from 'normal' and emphysematous lungs to respective ellipses is shown in Figure 2. Although the two groups overlapped, the bulk of specimens from normal lungs conformed reasonably well to their ellipses (corresponding to hypothetical planes through cylinders). In contrast, substantial spread was evident in bronchioles of lungs with unequivocal emphysema. These trends are presented in a different way in Fig. 3, using the mean conformity index of $85 \%$ for all bronchiolar samples as a reference. Seventy-four per

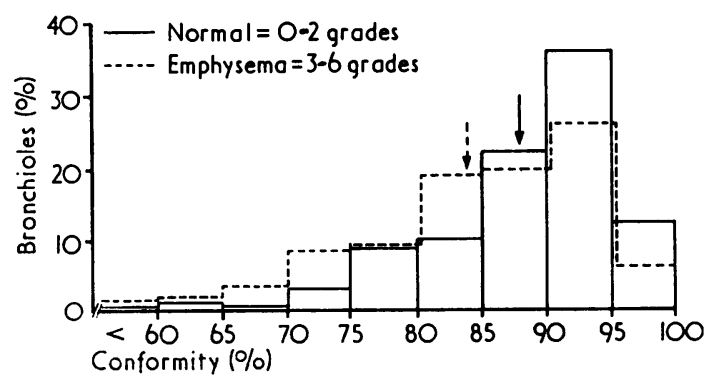

Fig. 2 Frequency distribution by percentage conformity of lumina of 140 nonrespiratory bronchioles from normal lungs and 234 bronchioles from emphysematous lungs to ellipses of same areas and circumferences. (None of the bronchioles of either group showed less than $50 \%$ conformity.)

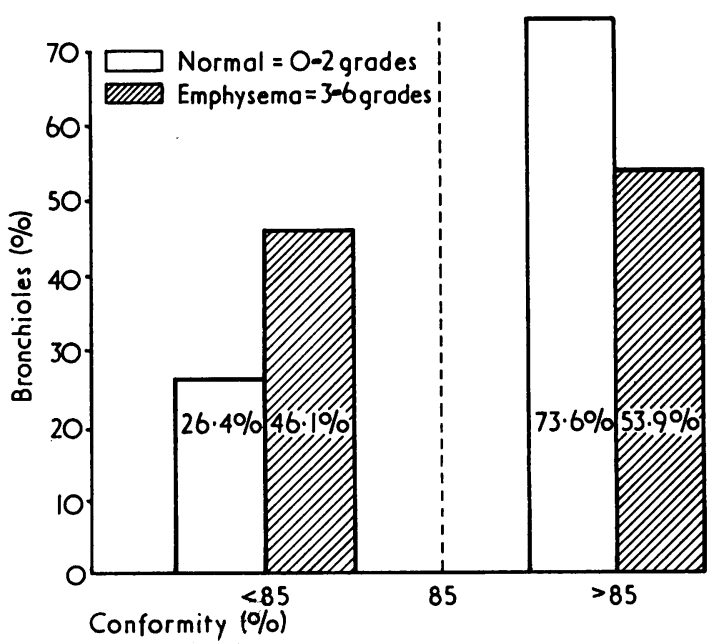

Fig. 3 Distribution of 140 nonrespiratory bronchioles from normal lungs and 234 nonrespiratory bronchioles from emphysematous lungs in relation to mean luminal conformity of $85 \%$ for all specimens. 
cent of bronchioles of normal lungs exceeded this level of conformity, whereas bronchioles from lungs with emphysema were approximately equally dispersed above and below this point $(P<0.001$ with $\chi^{2}$ test). The mean indices of bronchiolar conformity were $88 \%$ for the normal group and $84 \%$ for specimens with emphysema. Though not as striking as might be expected due to overlapping trends, these differences were significant ( $P<0.001$ with $t$ test). Figures 4 and 5 are replicas
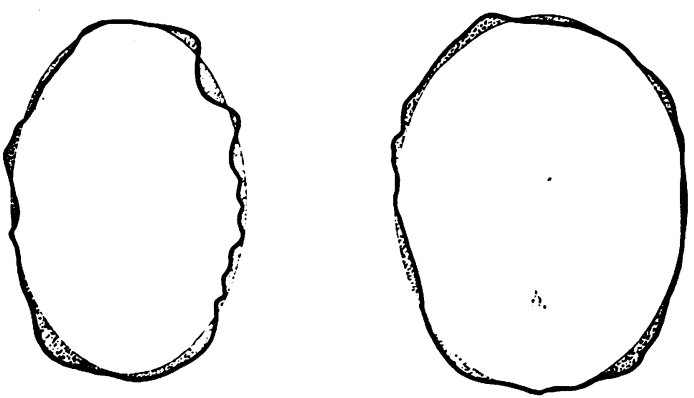

Fig. 4 Replicas of lumina of nonrespiratory bronchioles from histological sections showing instances of good conformity to respective ellipses of same areas and circumferences (over $98 \%$ ). Smaller specimen on left came from normal lung. Larger specimen on right was from a case of emphysema and was an exception to the general trend with respect to conformity for lungs with disease.
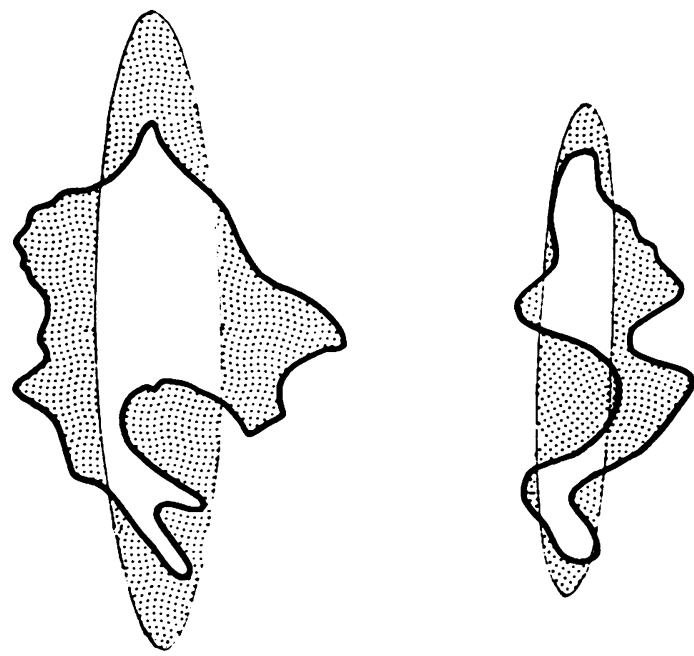

Fig. 5 Replicas of lumina of nonrespiratory bronchioles from histological sections of emphysematous lungs demonstrating poor conformity to ellipses of comparable areas and circumferences. Such specimens demonstrated a predilection for lungs with emphysema. of bronchiolar lumina and corresponding ellipses depicting some individual variations encountere

Distributions of nonrespiratory bronchioles $f$ essentially normal and emphysematous lungs afe shown by luminal diameter in Table 2 and lumingl area in Table 3. Both measures showed similar trends. Thus, $36 \%$ of bronchioles from lungs with emphysema had internal diameters of less that $0.2 \mathrm{~mm}$ compared to $10 \%$ of normal bronchioles ( $P<0.001$ with $\chi^{2}$ test). By area, $45 \%$ of the no respiratory bronchioles from lungs with emphi sema had lumina of under $0.10 \mathrm{~mm}^{2}$ and fewer than $20 \%$ of normal bronchioles were in this category $(\mathrm{P}<0.001)$. Deformed bronchioles occus red in airways of all sizes, but there was an especitally high incidence of deformity in the smallest species from lungs with disease. For example, of the 59 histological sections with a conformity index of less than $85 \%$ and an internal diameter of under $0.20 \mathrm{~mm}$ came from lungs with emphy sema $(\mathrm{P}<0.001)$. Fifty of the 53 sections with -9 conformity index of less than $85 \%$ and a luminal

Table 2 Distribution of nonrespiratory bronchioles from normal and emplyysematous lungs by luminal diameter

\begin{tabular}{|c|c|c|c|c|c|}
\hline \multirow{2}{*}{$\begin{array}{l}\text { Bronchiolar } \\
\text { luminal } \\
\text { diameter } \\
(\mathrm{mm})\end{array}$} & \multicolumn{2}{|c|}{$\begin{array}{l}\text { Normal } \\
(0-2)\end{array}$} & \multicolumn{2}{|c|}{$\begin{array}{l}\text { Emphysema } \\
(3-6)\end{array}$} & \multirow{2}{*}{$\frac{\text { Total }}{\text { No. }}$} \\
\hline & No. & $\%$ & No. & $\%$ & \\
\hline $0-0.19$ & 14 & 10 & 83 & 36 & 97 \\
\hline $0.20-0.39$ & 71 & 51 & 87 & 37 & 158 \\
\hline $0.40-0.59$ & 34 & 24 & 35 & 15 & 69 \\
\hline $0.60-0.79$ & 11 & 8 & 18 & 8 & 29 \\
\hline $0.80-0.99$ & 6 & 4 & 4 & 2 & 10 \\
\hline $1 \cdot 00-1 \cdot 19$ & 2 & 2 & 3 & 1 & 5 \\
\hline $1 \cdot 20-1 \cdot 39$ & 2 & 1 & 1 & - & 3 \\
\hline $1 \cdot 40-1 \cdot 59$ & - & - & 2 & 1 & 2 \\
\hline $1 \cdot 60-1 \cdot 79$ & - & - & - & - & - \\
\hline $1 \cdot 80+$ & - & - & 1 & - & 1 \\
\hline Total & 140 & 100 & 234 & 100 & 374 \\
\hline
\end{tabular}

Table 3 Distribution of nonrespiratory bronchioles from normal and emphysematous lungs by luminal area

\begin{tabular}{|c|c|c|c|c|c|}
\hline \multirow{2}{*}{$\begin{array}{l}\text { Bronchiolar } \\
\text { luminal } \\
\text { area } \\
\left(\mathrm{mm}^{2}\right)\end{array}$} & \multicolumn{2}{|c|}{$\begin{array}{l}\text { Normal } \\
(0-2)\end{array}$} & \multicolumn{2}{|c|}{$\begin{array}{l}\text { Emphysema } \\
(3-6)\end{array}$} & \multirow{2}{*}{$\frac{\text { Total }}{\text { No. }}$} \\
\hline & No. & $\%$ & No. & $\%$ & \\
\hline $\begin{array}{l}0-0.09 \\
0.10-0.19 \\
0 \cdot 20-0.29 \\
0.30-0.39 \\
0.40-0.49 \\
0.50-0.59 \\
0.60-0.69 \\
0.70+\end{array}$ & $\begin{array}{r}28 \\
43 \\
23 \\
15 \\
7 \\
6 \\
9 \\
9\end{array}$ & $\begin{array}{r}20 \\
31 \\
17 \\
11 \\
5 \\
4 \\
6 \\
6\end{array}$ & $\begin{array}{r}106 \\
47 \\
30 \\
18 \\
4 \\
6 \\
3 \\
20\end{array}$ & $\begin{array}{r}45 \\
20 \\
13 \\
8 \\
2 \\
3 \\
1 \\
8\end{array}$ & $\begin{array}{r}134 \\
90 \\
53 \\
33 \\
11 \\
12 \\
12 \\
29\end{array}$ \\
\hline Total & 140 & 100 & 234 & 100 & 374 \\
\hline
\end{tabular}


AREA
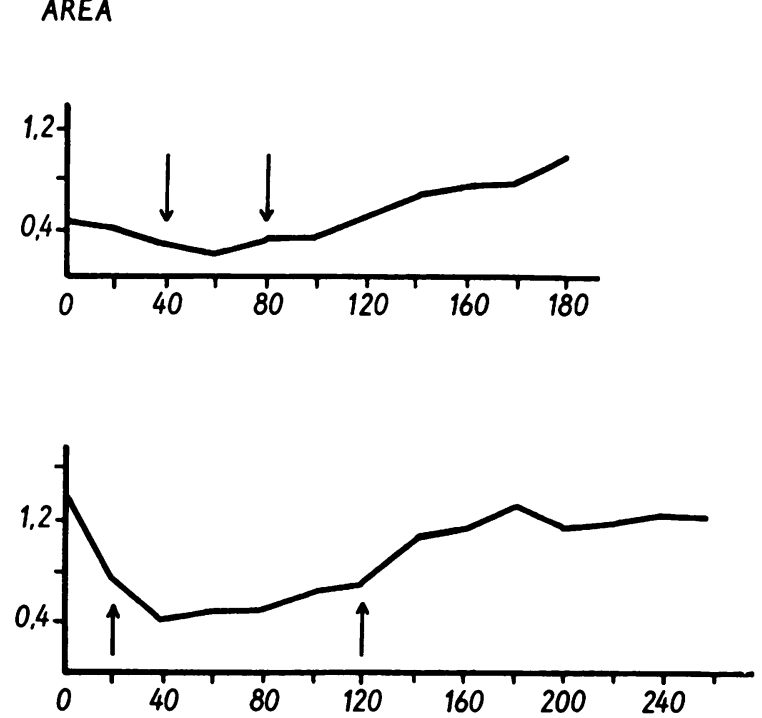

AREA

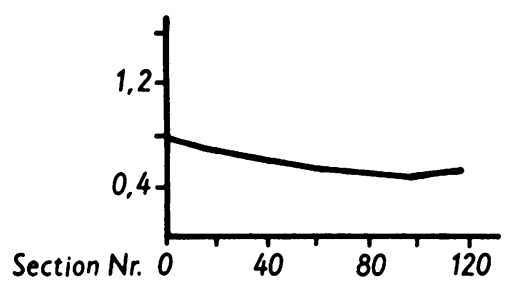

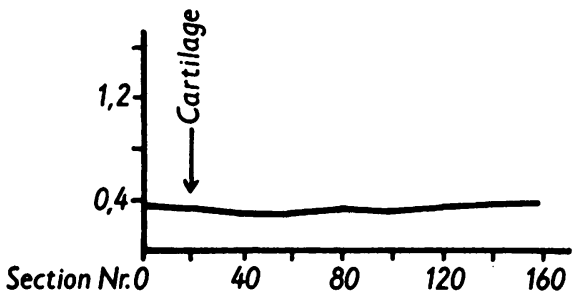

Fig. 6 Relation between luminal area and longitudinal level of nonrespiratory bronchioles from normal and emphysematous lungs. Area is shown in square millimetres and section numbers correspond to increments of $120 \mathrm{~m} \mu$ along bronchiolar long axes. The two specimens from normal subjects on left demonstrate only minor variations in area from level to level. In contrast, there are wide fluctuations in luminal area by air passage level in the narrowed bronchioles from emphysematous subjects on the right (extent of stenoses roughly indicated by arrows).

area of less than $0.10 \mathrm{~mm}^{2}$ were from emphysematous sections $(P<0.001)$.

Nonrespiratory bronchioles from emphysema also tended to be irregular in a longitudinal direction. This may be appreciated in Fig. 6 which shows relations between luminal area and longitudinal level of segments of bronchioles from normal and emphysematous lungs. The normal specimens on the left are relatively uniform in calibre. In the samples from emphysema on the right, pronounced fluctuations in cross-sectional area occur along the longitudinal axes. The extent of stenoses is indicated by arrows. A comparison of areas at stenotic levels such as this with that of the respective bronchioles in general is shown in Fig. 7 as per cent deviation from mean area. Deviations of 20 to $70 \%$ were characteristic of the 15 small air passages in emphysema; however, only one of the 14 normal specimens differed by more than $30 \%$ from the mean for all segments in that channel.

\section{Discussion}

Some potential sources of error in our methods are worthy of note. All lung specimens were inflation-fixed as recommended by Gough and Wentworth (1960), and Matsuba and Thurlbeck (1971)

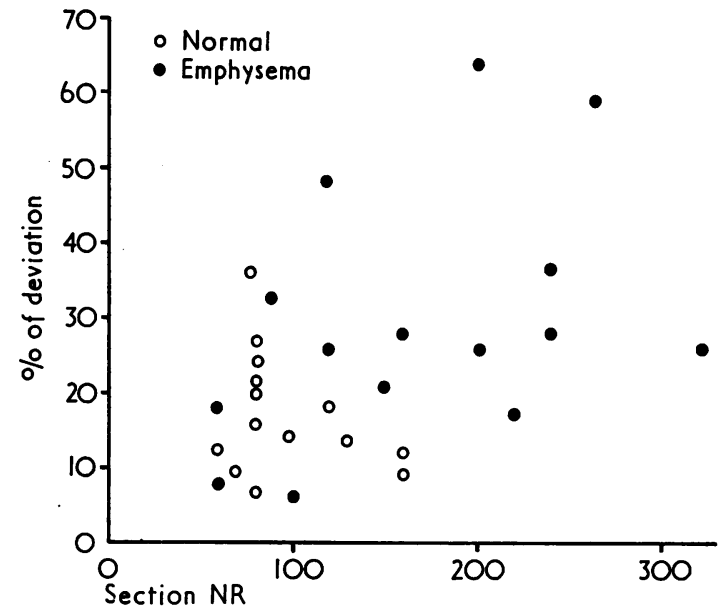

Fig. 7 Variation between luminal area of smallest level and mean area of all levels at regular increments (see Fig. 6) for 14 nonrespiratory (NR) bronchioles from normal lungs and 15 nonrespiratory bronchioles from emphysematous lungs. Deviations are much more pronounced in bronchioles from emphysema than in normal samples.

observed that this may lead to inaccuracies through improper expansion and shrinkage during 
fixation. In our experience, such variation has seemed comparatively small (less than $10 \%$ difference between final and 'true' inspiratory relationships (Anderson et al., 1964) and should not, in any event, alter shapes and relative trends seriously. Superimposition of ellipses and bronchiolar replicas involved a certain element of subjectivity. When doubts existed, several possible positions were tried and that first chosen usually produced the closest conformity.

The lumina of histological preparations of most nonrespiratory bronchioles from normal lungs appeared to conform closely to ellipses of comparable areas and circumferences, thus corroborating the original premise of this study. Such ellipses, by definition, reflect sections of varying obliquity through cylinders. Although some of the sections from emphysematous lungs also conformed well, deviations from the ellipsoid pattern occurred much more often than expected. Lumen diameter and area in emphysema also differed from that in normal specimens. Thus, lungs with disease had fewer intermediate sized nonrespiratory bronchioles and an excess of extremely small air passages. These observations are in agreement with previous data reported from our laboratories (Linhartová et al., 1971) and are similar to the trend observed by Matsuba and Thurlbeck (1972). Plane section deformities occurred in bronchioles of all sizes in the present investigation, but they were particularly common in the smallest variety, the one most frequent in emphysema. This feature seemed, furthermore, to have three-dimensional expression, for the lumina of stenotic bronchioles in emphysema were irregular in a longitudinal direction. Two earlier studies from our laboratories, one based on a somewhat similar but less detailed methodological concept (Linhartová et al., 1973) and the other on restricted sampling (Linhartová et al., 1974), also showed an association between luminal deformities and bronchiolar stenosis. It thus seems reasonable to conclude that deformity in luminal contours is an important factor in the characteristic stenosis of the most distal conducting airways of lungs with centrilobular emphysema.

Although the ellipsoid shape, and by inference a cylindroid configuration, was typical of the most peripheral conducting air passages of normal lungs, the lumina of some of these seemed deformed. This could not be attributed to oblique histological sectioning, since the methodólogy was designed to compensate for this variation. Moreover, potential distortions associated with inflationfixation errors are thought to be relatively small, as mentioned above. Two possibilities whieh seemed more likely in our opinion include ina vertent inclusion of irregular sections adjacent bronchiolar divisions and the normal variations which characterise almost all anatomical systems. On the other hand, the excessive incidence of deformity in the nonrespiratory bronchioles from centrilobular emphysema is considered to be $\overrightarrow{a m}$ intrinsic feature of the disease itself. Causatipe mechanisms have been dealt with in some detat in earlier communications (Linhartová et alu 1971; Linhartová et al., 1973; Linhartová et dut. 1974; Anderson and Foraker, 1974). Briefly, the most important single factor is thought to be log్s of radial tractional support or 'lung tension' (Dayman, 1951) secondary to rupture of the peribro $\overrightarrow{\eta_{-}}$ chiolar alveolar walls in disruptive pulmonafy emphysema. This in turn permits collapse and narrowing of the lumina of the semimembranou contractile, nonrespiratory bronchioles. Since centrilobular emphysema is typically focal in dist tribution (CIBA Guest Symposium, 1959; American Thoracic Society, 1962), some bronchiolgs may be spared. When involvement does occur, sुt may be in a segmental fashion. Airflow restriction and turbulence are then logical consequences.

\section{References}

American Thoracic Scciety (1962). Definitions a凷 classification of chronic bronchitis, asthma, aरिd pulmonary emphysema. American Review की Respiratory Diseases, 85, 762-768.

Anderson, A. E. Jr. and Foraker, A. G. (1962). Re tive dimensions of bronchioles and parenchymal spaces in lungs from normal subjects and empł:sematous patients. American Journal of Medicinge, 32, 218-226.

Anderson, A. E. Jr. and Foraker, A. G. (1974). The nonrespiratory bronchioles in pulmonary emphysema. Pathology Annual, 9, 231-261.

Anderson, W. F., Anderson, A. E., Jr., Hernande J. A., and Foraker, A. G. (1964). Topography rof aging and emphysematous lungs. American Rev.ew of Respiratory Diseases, 90, 411-423.

ก

Campbell, E. J. M., Martin, H. B., and Riley, R. T్. (1957). Mechanisms of airway obstruction.' Bulleț of the Johns Hopkins Hospital, 101, 329-343.

CIBA Guest Symposium (1959). Terminology, definitions, and classification of chronic pulmonfofy emphysema and related conditions. Thorax, 14, 286299.

Dayman, H. (1951). Mechanics of airflow in hea and in emphysema. Journal of Clinical Inyestigation. 30, 1175-1190.

Depierre, A., Bignon, J., Lebeau, A., and Brouet, $\frac{O}{\mathcal{F}}$. (1972). Quantitative study of parenchyma and smâH conductive airways in chronic nonspecific luagg 
disease: use of histologic stereology and bronchial casts. Chest, 62, 699-708.

Gough, J. and Wentworth, J. E. (1960). Thin sections of entire organs mounted on paper. In Recent Advances in Pathology, 7th edition, p. 80. Edited by C. V. Harrison. Churchill, London.

Hogg, J. C., Macklem, P. T., and Thurlbeck, W. M. (1968). Site and nature of airway obstruction in chronic obstructive lung disease. New England Journal of Medicine, 278, 1355-1360.

Linhartová, A., Anderson, A. E., Jr., and Foraker, A. G. (1971). Radial traction and bronchiolar obstruction in pulmonary emphysema: observed and theoretical aspects. Archives of Pathology, 92, 384-391.

Linhartová, A., Anderson, A. E., and Foraker, A. G. (1973). Nonrespiratory bronchiolar deformities: graphic assessment in normal and emphysematous lungs. Archives of Pathology, 95, 45-47.

Linhartová, A., Anderson, A. E., Jr., and Foraker, A. G. (1974). Topology of nonrespiratory bronchi- oles in normal and emphysematous lungs. Human Pathology, 5, 729-735.

Matsuba, K. and Thurlbeck, W. M. (1971). The number and dimensions of small airways in nonemphysematous lungs. American Review of Respiratory Disease, 104, 516-524.

Matsuba, K. and Thurlbeck, W. M. (1972). The number and dimensions of small airways in emphysematous lungs. American Journal of Pathology, 67, 265-275.

Silvers, G. W., Maisel, J. C., Petty, T. L., Mitchell, R. S., and Filley G. F. (1972). Central airway resistance in excised emphysematous lungs. Chest, 61, 603-612.

Spain, D. M. and Kaufman, G. (1953). The basic lesion in chronic pulmonary emphysema. American Review of Tuberculosis, 68, 24-30.

Requests for reprints to: Dr. A. E. Anderson, Research Department, Baptist Memorial Hospital, 800 Prudential Drive, Jacksonville, Florida, 32207, USA. 